\title{
ANALYSIS OF LEARNING FRAMEWORK FOR PERSONALIZED DIVERSIFICATION
}

\author{
${ }^{1}$ A. Uthiramoorthy, ${ }^{2}$ Dr. R. Muralidharan \\ ${ }^{1}$ Research Scholar, Department of Computer Science, Rathinam College of Arts \& Science, Coimbatore. \\ ${ }^{2}$ Principal, Rathinam College of Arts \& Science, Coimbatore.
}

\section{ABSTRACT}

The Web is rapidly growing in length, and it has grown to be the most crucial commodity for the society to get access to, create, and distribute widespread facts. Nonetheless, finding relevant information from this massive repertoire of content material isn't constantly clean for beginners. Personalized web search (PWS) is a standard class of search strategies aiming at imparting better search effects, which can be tailored for user needs. As the expense, user statistics must be accrued and analyzed to discern out the user aim in the back of the issued query. Personalized search is an important constituent of a search engine since it enables in enhancing outcomes based at the user's recorded pastimes.

Search result diversification has received attention as a method to tackle query ambiguity. In search result diversification one usually considers the relevance of a document in light of the alternative retrieved documents. The aim is to become aware of the possibly "factors" of the ambiguous query, retrieve files for each of these factors, and make the quest affects more various.

Several tactics addressed these troubles inside the unsupervised learning knowledge of way. But it produces a whole lot of pitfalls or set back within the evaluation of performance. This proposed approach also addresses the problem personalised diversification of search results to beautify both diversification and personalization performances. To in addition, enhance performance, a supervised learning knowledge of approach is proposed.

Key words: - PWS, Diversification, Framework, Query, Profile, Machine Learning.

\section{INTRODUCTION}

Current web search engines like Google and yahoo are constructed to serve all customers, independent of the special needs of any character user. With the exponential boom of the to be had facts at the World Wide Web, a conventional search engine, despite the fact that primarily based on state-of-the-art report indexing algorithms, has issue meeting performance and effectiveness performance demanded by means of customers attempting to find relevant statistics. The personalization of web search is to carry out retrieval for each user incorporating his/her pursuits. Personalized web search differs from established web search, which returns identical results to all users for identical queries, regardless of various user hobbies and facts needs. When queries are issued to search engines, maximum go back the identical effects to customers. The considerable majority of queries to search engines like Google are short and ambiguous. Different customers may also have completely exclusive statistics wishes and goals whilst using precisely the identical question [8].

Personalized web search may be executed via checking content material similarity between web pages and user profiles. Some work has represented user pursuits with topical classes. User's topical hobbies are either explicitly detailed through users themselves or can be robotically found out by classifying implicit user records. Search effects are filtered or re-ranked with the aid of checking the similarity of subjects among search results and user profiles [10].

Most usual web search tactics to rank the significance of files based totally at the linkage shape of the web. An intuitive method to personalised web search is to evolve those algorithms to compute personalized significance of documents [8].

There are two most important components inside the proposed framework, referred to as, personalised web search and search result diversification, which play important roles in tackling the problem of personalised search result diversification. The task of personalised web search aims at identifying the maximum relevant search results for an character via leveraging their data [11]. Many personalized web search methods have been proposed, consisting of the one based totally on social tagging profiles, ranking version adaption for personalized search, search personalization via modelling the effect of customers' behavior, and personalized search using interplay behaviors in search sessions.

The existing method addressed these two issues inside the unsupervised mastering way. But it produces quite a few pitfalls or set back inside the assessment of overall performance [12].

\section{RELATED WORK}

Though the Personalization of user information is a recent phenomenon because of the upward push of Web utilization, through the years Web mining techniques have developed fully-fledged assist to provide a personalized revel in for customers. A lot of labor is finished to improvise Web Personalization and diversification [9].

Personalized search is an important way to improve the overall performance of a search engine. In this approach, 
proposed a framework that helps mining a user's conceptual alternatives from customers' clickthrough data due to Web search. The found possibilities are applied to adapt a search engine's rating function. In this framework, an extended set of conceptual preferences changed into derived for a user based totally on the concepts extracted from the quest results and the clickthrough statistics. Then, a idea-primarily based user profile (CUP) representing the user profile as a concept ontology tree is generated. Finally, the CUP is input to a guide vector system (SVM) to research a concept choice vector for adapting a personalized rating characteristic that re-ranks the search results. To attain extra bendy personalization, the framework lets in a user to control the amount of precise CUP ontology records to be uncovered to the personalized search engine. Further, observed numerous parameters, which includes conceptual relationships and concept capabilities, bobbing up from a CUP that influences the ranking great. Experiments verify that our approach can appreciably improve the retrieval effectiveness for the user. [1].

Search result diversification targets to retrieve numerous results to fulfill as many specific facts needs as possible. Supervised techniques have been proposed recently to study rating capabilities and that they have been proven to supply advanced results to unsupervised strategies. However, these strategies use implicit approaches based totally on the precept of Maximal Marginal Relevance (MMR). In this approach, getting to know framework for express result diversification wherein subtopics are explicitly modeled. Based at the statistics contained within the sequence of decided on documents, to use attention mechanisms to capture the subtopics to be centered on even as selecting the subsequent document, which obviously fits our project of report selection for diversification. As an initial attempt, and rent recurrent neural networks and maxpooling to instantiate the framework. To use each disbursed representations and traditional relevance capabilities to model documents within the implementation. The framework is flexible to model question motive in either a flat list or a hierarchy. [2].

Query hints assist users refine their queries after they input an preliminary query. Previous work on query thought has specifically concentrated on strategies that are similarity-based totally or context-primarily based, developing fashions that both cognizance on adapting to a specific user (personalization) or on diversifying query elements to maximize the opportunity of the user being glad (diversification). It is difficult to forget the undertaking of producing query suggestions that are both personalised and different. To suggest a customised query inspiration diversification (PQSD) model, wherein a user's lengthy-term search behavior is injected right into a simple grasping query proposal diversification model that considers a user's search context in their modern-day session. Query aspects are diagnosed via clicked documents based on the Open Directory Project (ODP) with a Latent Dirichlet Allocation (LDA) subject matter model. Then quantify the improvement of our proposed PQSD model against a stateof-the-art baseline the use of the general public America Online (AOL) question $\log$ and show that it beats the baseline in phrases of metrics utilized in question suggestion ranking and diversification [3].

A multi-project learning knowledge of framework to together research record ranking and query concept for web search. It consists of two primary components, a report ranker, and a query recommender. Document ranker combines contemporary query and consultation information and compares the mixed representation with document representation to rank the files. Query recommender tracks customers' question reformulation series thinking about all previous in-session queries using a series to sequence technique. As each responsibilities are driven through the users' underlying search rationale, to perform joint learning knowledge of these components through consultation recurrence, which encodes search context and cause. Extensive comparisons in opposition to latest report ranking and query notion algorithms are done on the general public AOL search log, and the promising results advise the effectiveness of the joint mastering framework. [4].

The trouble of personalized diversification of search effects, to decorate the performance of both undeniable diversification and undeniable personalization algorithms. In preceding work, the trouble has specifically been tackled thru unsupervised getting to know. In addition beautify the performance, and to advocate a supervised studying strategy. Specifically, install an established learning framework for accomplishing supervised personalized diversification, in which to upload functions extracted at once from tokens of documents and those used by unsupervised personalized diversification algorithms, and, importantly, those generated from our proposed userhobby latent Dirichlet topic model. Additionally define constraints in our established getting to know framework to ensure that search outcomes are both different and consistent with a user's hobby. To in addition increase the performance of schooling, propose a fast training framework for our proposed approach by using adding additional more than one particularly violated however also diverse constraints at every education iteration of the cutting-aircraft set of rules. To experiments on an open dataset and locate that our supervised learning knowledge of strategy outperforms unsupervised personalized diversification methods as well as different simple personalization and simple diversification methods. [5].

The trouble of answering ambiguous web queries in a setting in which there exists taxonomy of statistics, and that each queries and files may also belong to more than one class in step with this taxonomy. Here, present a scientific technique to diversifying results that intention to minimize the hazard of dissatisfaction of the common user. Here, propose a set of rules that well approximates this goal in widespread, and is probably choicest for a herbal unique case. Furthermore, generalize numerous classical IR metrics, along with NDCG, MRR, and MAP, to explicitly account for the fee of diversification. To show empirically that our set of rules scores better in those generalized metrics in comparison to results produced through industrial engines like Google. [6].

A unique method designed to balance and diversify personalized recommendation lists to mirror the user's whole spectrum of pursuits. Though being unfavourable to average accuracy, show that our technique improves user satisfaction with advice lists, in particular for lists generated using the commonplace item-based collaborative filtering algorithm. Our work builds upon earlier research on recommender structures, looking at properties of recommendation lists as entities of their personal right instead of in particular focusing at the accuracy of character guidelines. To 
introduce the intra-listing similarity metric to evaluate the topical range of advice lists and the subject diversification method for lowering the intra-list similarity [7].

There is a awesome loss of studies that focus on the examination of performance troubles associated with modes of diversification - until currently. Although this is the first review of the literature that has simultaneously considered triggers and choice influencers for kind, mode, and level of diversification in a unmarried framework to probe the impact on company overall performance, nevertheless have sufficient proof from the integration of beyond research to finish that diversification selections have to be studied simultaneously.

\section{PROPOSED FRAMEWORK \& METHODOLOGY}

The problem of personalised diversification of search results is important to enhance the performance of the user query [13]. Previous paintings on question inspiration specially make a speciality of recommending semantically related queries in response to a user's enter query. Such strategies cannot handle queries with unsure search aspects, in particular for customers with diverse search intents. To alleviate the aforementioned trouble, two categories of procedures have been added to supplement conventional question proposal methods: diversification and personalization. In both search result diversification and personalized web search, an issued question is often viewed as an incomplete expression of a user's underlying need. Unlike search result diversification, where the device accepts and adapts its behavior to a state of affairs of uncertainty, personalized web search strives to address this example by using improving the device's know-how about users' statistics wishes.

Search results generated by using diversification techniques ought to be extra diverse when a user's options are unrelated to the query. Likewise, personalization can improve the effectiveness of factor weighing in diversification, by favouring question interpretations which can be anticipated to be greater associated with every particular user. To further, take the advantages of both personalization and diversification to recommend a personalized question proposal diversification version (PQDM), wherein diversification helps to generate a couple of-element queries to increase the probability of cautioned queries being clicked and personalization ensures that the advised queries are near a user's particular search reason. The proposed PQDM consists of two major stages.

$>$ In the first stage, to develop a greedy query notion diversification model (G-QDM) wherein a user's search context, which includes queries and clicks, is considered to generate a varied ranked listing of queries; to this case, and use co-occurrences in addition to the semantic similarity among queries.

$>$ In the second one level, to similarly, inject a user's long-time period search conduct information into the model proposed within the first step with Bayes' rule. And collect files that had been shown and clicked in response to a question primarily based on the search logs. Then, the incorporate supervised categorised latent Dirichlet allocation (LDA) to version the topic distribution of file descriptions.

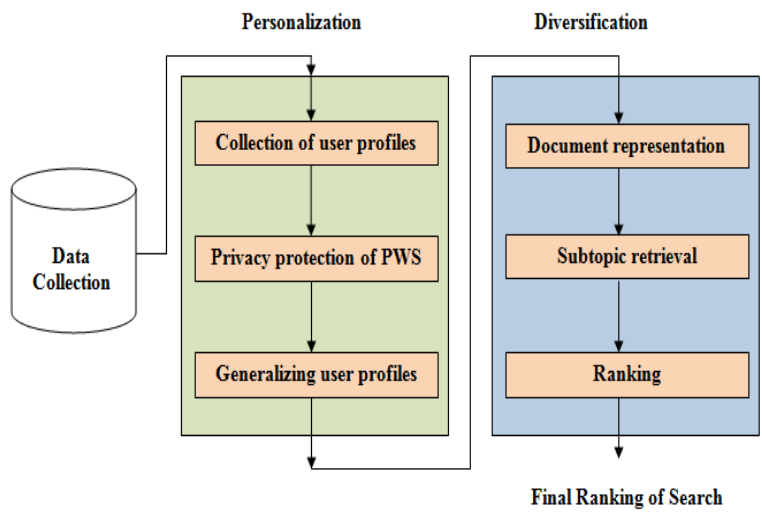

Figure 1: - Proposed Framework

Figure 1, shows the framework and structure of the proposed work and its phases. The proposed approach of personalized search results diversification as a problem of predicting a numerous set of documents is given a selected user and query. The main idea is to apply a Document Sequence with Subtopic Attention (DSSA) framework, from which to be able to infer an in line with-record multinomial distribution over topics and decide whether a report can cater to a particular user.

\section{Phase 1: - Personalized Search}

Personalized search is an essential constituent of a search engine since it allows in enhancing results based at the user's recorded pursuits. Not simplest past search queries, however past clicked effects may be used as implicit behavior for improvement of results by means of boosting the clicked results. Another interesting element of personalization is the use of question expansion through mining user logs.

\section{$>$ Profile-Based Personalization}

This paper introduces an approach to personalizing virtual multimedia content material based totally on user profile data. For this, two important mechanisms had been developed: a profile generator that robotically creates user profiles representing the user alternatives, and a content-based recommendation algorithm that estimates the user's hobby in unknown content material by matching her profile to metadata descriptions of the content. Both capabilities are integrated right into a personalization device.

\section{$>$ Privacy Protection in PWS System}

To advise a PWS framework called UPS that may generalize profiles in for each query in line with userexact privacy necessities. Two predictive metrics are proposed to assess the privacy breach risk and the query application for a hierarchical user profile. Here, to develop a simple however powerful generalization algorithms for user profiles taking into account question-degree customization the usage of our proposed metrics. Additionally provide an online prediction mechanism primarily based on query utility for identifying whether or not to personalize a query in UPS. Extensive experiments exhibit the performance and effectiveness of our framework.

\section{$>$ Generalizing User Profile}

The generalization system has to fulfil specific stipulations to address the user profile. This is achieved with the aid of pre-processing the user profile. At first, the system initializes the user profile by taking the indicated discern user profile into consideration. The 
manner provides the inherited residences to the homes of the neighborhood user profile. Thereafter the procedure loads the facts for the foreground and the background of the map in step with the described choice inside the user profile.

\section{Phase 2: - Diversification of Search}

Diversity plays an important role in modern search engines as information on the World Wide Web (WWW) has increased manifold, users don't prefer to view similar information. Also, there are lots of queries on web search which are ambiguous. Traditional approaches to search result diversification are usually unsupervised and adopt manually defined functions with empirically tuned parameters. Depending on whether the underlying intents (or subtopics) are explicitly modelled, they can be categorized into implicit and explicit approaches. To avoid heuristic and handcrafted functions and parameters, a new family of research work using supervised learning is proposed.

\section{$>$ Document Sequence Representation}

The representation of a document is composed of two parts: distributed representations and relevance features. The distributed representation can be constructed in different ways. Relevance features are those used in traditional IR, such as BM25 score, etc.

\section{$>$ Subtopic Retrieval}

Subtopic retrieval problem in information retrieval poses a different challenge compared to normal retrieval problems. it ensures that the retrieved results contain all perspectives of the query. Modern search engines attempt to resolve ambiguity by auto-suggesting queries to the end-users based on query logs. The proposed system using the Document Sequence with Subtopic Attention Framework (DSSA). To further, instantiate DSSA to a concrete form and articulate the training and prediction algorithms. The main idea of DSSA is to dynamically capture accumulative relevance information of previous document sequence, to calculate subtopic attention.

\section{$>$ Scoring (or) Ranking}

The final part of the diversification is scoring. The range rating is calculated as a weighted aggregate of the document's relevance to each subtopic with the aid of interest distribution. To use the equal way to calculate the file's relevance to a question and its subtopics the usage of each distributional representations and relevance capabilities, even though extraordinary ways may be used.

Personalization components are associated with the individual user whilst diversification aims only to satisfy the most viable wide variety of users. The advantages of the proposed framework are as follows,

$>$ The problem of personalised diversification of search results in a brand new way, using a supervised getting to know technique.

$>$ It affords runtime profiling, which in effect optimizes the personalization utility at the same time as respecting user's privateness necessities;

$>$ A categorised latent topic model to according torecord multinomial distributions over topics.

$>$ Further, it boosts the performance of the schooling degree of our approach by including no longer simplest relatively violated but additionally various constraints at some point of schooling iterations.

\subsection{Study of the existing system}

In previous paintings, the problem has specially been tackled through unsupervised mastering. In this approach, the styles are semantically that means complete text and are acquired via searching within the retrieved result set. In comparison, profile-based strategies enhance the search experience with complicated user-interest models generated from user profiling strategies [14]. Profile-based totally techniques may be doubtlessly powerful for nearly all kinds of queries however are stated to be risky underneath some situations. A user profile is generally generalized only as soon as offline and used to personalize all queries from the equal user indiscriminatingly. Such a "one profile suits all" method clearly has drawbacks given the form of queries [5]. One evidence stated is that profile-primarily based personalization may not even help to improve the quest firstrate for a few ad hoc queries, although exposing user profile to a server has placed the user's privateness at danger. Then, from the candidate patterns that means complete applicants are chosen as subtopics based on unique methods like IDF (Inverse Document Frequency), Term importance rating, etc. Another interesting work inside the current technique presented with the aid of the use of MMR based approach, also incorporating novelty with variety for subtopic retrieval.

The shortcomings of the prevailing method are as follows,

$>$ The current profile-based PWS does not assist runtime Profiling.

$>$ The existing methods do no longer recall the customization of privateness necessities.

$>$ Many personalization techniques require iterative user interactions when growing personalized search outcomes.

\section{PERFORMANCE EVALUATION METRICS}

The following overall performance parameters are typically used in search result Personalization and diversification assessment [14]. The current technique is compared with the proposed scheme the use of these assessment parameters.

\subsection{Recall and Precision}

Precision can be seen as a degree of exactness or pleasant, whereas recollect is a degree of completeness or quantity. In easy terms, excessive precision approach that an set of rules lower back considerably more relevant results than beside the point ones, while high don't forget manner that an set of rules lower back most of the relevant results.

$$
\begin{aligned}
& \text { Precision }=\frac{\text { No. of correct results }}{\text { No. of all returned results }} \\
& \text { Recall }=\frac{\text { No. of correct results }}{\text { total number of actual results }}
\end{aligned}
$$

\subsection{F-Measure}

F-degree combines precision and keeps in mind and is the harmonic suggest of precision and recollect. 


$$
F-\text { measure }=2 \times \frac{\text { precision } x \text { recall }}{\text { precision }+ \text { recall }}
$$

Several experiments were performed with the oneof-a-kind query and the precision, bear in mind, and Fdegree of the output was calculated. This better improvement in precision value can compromise for the very small percent of a drop within the bear in mind cost. Moreover, the F-degree which combines precision and keep in mind is much progressed for similarity than the present machine.

\subsection{Eleven-Point average Precision}

It is a degree primarily based on remember and precision. Given a record, the machine is allowed to acquire recall values of $0 \%, 10 \%, 20 \%$. . A hundred $\%$ and the precision values at those points are computed. The resulting eleven point precision values are then averaged. The common precision values of all take a look at documents are further averaged to acquire a international degree of the device overall performance.

\subsection{Break-Even Point}

It is the point where recall equals precision. It is acquired by permitting the classifier to assign greater categories. As a result, the remember increases and precision decreases until they become equal.

\section{CONCLUSION}

The awesome improvement of facts at the Web has forced new challenges for the construction of effective search engines. This studies painting presents a singular method to go looking result personalization and diversification. In maximum previous work on personalized Web search, all queries had been usually personalised in the same way. The proposed framework introduces the user interest-primarily based subject matter model to seize user pastimes, Based in this, several user interest capabilities are extracted and the similarity between a user and a document may be successfully measured for our studying approach. The two proposed constraints are shown to play a substantial role inside the supervised method. It also discovers that the user-interest subject matter version enables to improve performance. Our proposed getting to know technique can go back greater subtopics.

\section{REFERENCES}

[1] Leung, K. W. T., Lee, D. L., Ng, W., \& Fung, H. Y. (2008). A framework for personalizing web search with concept-based user profiles. ACM Transactions on Internet Technology (TOIT), 11(4), 1-29.

[2] Jiang, Z., Dou, Z., Zhao, W. X., Nie, J. Y., Yue, M., \& Wen, J. R. (2018). Supervised search result diversification via subtopic attention. IEEE Transactions on Knowledge and Data Engineering, 30(10), 1971-1984.

[3] Chen, W., Cai, F., Chen, H., \& de Rijke, M. (2017). Personalized query suggestion diversification. In Proceedings of the 40th International ACM
SIGIR Conference on Research and Development in Information Retrieval (pp. 817-820).

[4] Ahmad, W. U., Chang, K. W., \& Wang, H. (2018). Multi-task learning for document ranking and query suggestion. In International Conference on Learning Representations.

[5] Liang, S., Cai, F., Ren, Z., \& de Rijke, M. (2016). Efficient structured learning for personalized diversification. IEEE Transactions on Knowledge and Data Engineering, 28(11), 2958-2973.

[6] Agrawal, R., Gollapudi, S., Halverson, A., \& Ieong, S. (2009). Diversifying search results. In Proceedings of the second ACM international conference on web search and data mining (pp. 514).

[7] Ziegler, C. N., McNee, S. M., Konstan, J. A., \& Lausen, G. (2005). Improving recommendation lists through topic diversification. In Proceedings of the 14th international conference on World Wide Web (pp. 22-32).

[8] Joshi, C., Jaiswal, T., \& Gaur, H. (2013). An overview study of personalized web search. International Journal of Scientific and Research Publications, 3(1).

[9] Li, W., Wang, X., Hu, R., \& Tian, J. (2011). User interest modeling by labeled LDA with topic features. In 2011 IEEE International Conference on Cloud Computing and Intelligence Systems (pp. 6-11). IEEE.

[10] Liang, S., Ren, Z., \& De Rijke, M. (2014). Personalized search result diversification via structured learning. In Proceedings of the 20th $A C M$ SIGKDD international conference on Knowledge discovery and data mining (pp. 751760).

[11] Ahmad, W. U., Chang, K. W., \& Wang, H. (2018). Multi-task learning for document ranking and query suggestion. In International Conference on Learning Representations.

[12] Zhao, Z., Cheng, Z., Hong, L., \& Chi, E. H. (2015). Improving user topic interest profiles by behavior factorization. In Proceedings of the 24th International Conference on World Wide Web (pp. 1406-1416).

[13] Mcauliffe, J. D., \& Blei, D. M. (2008). Supervised topic models. In Advances in neural information processing systems (pp. 121-128).

[14] Vallet, D., \& Castells, P. (2012). Personalized diversification of search results. In Proceedings of the 35th international ACM SIGIR conference on Research and development in information retrieval (pp. 841-850). 